\title{
Diffusion Tensor Imaging of the Brain
}

\author{
Andrew L. Alexander, ${ }^{* *}$ Jee Eun Lee, ${ }^{*}$ Mariana Lazar, ${ }^{\ddagger I I}$ and Aaron S. Field ${ }^{\S \|}$ \\ *Departments of Medical Physics, ${ }^{\dagger}$ Psychiatry, ${ }^{\circledR}$ Radiology, and ${ }^{\|}$Biomedical Engineering and the ${ }^{\ddagger}$ Waisman Laboratory for \\ Brain Imaging and Behavior, Waisman Center, University of Wisconsin-Madison, Madison, Wisconsin 53706; and the ${ }^{\text {II }}$ Center \\ for Biomedical Imaging, Radiology Department, New York University School of Medicine, New York, New York 10016
}

\begin{abstract}
Summary: Diffusion tensor imaging (DTI) is a promising method for characterizing microstructural changes or differences with neuropathology and treatment. The diffusion tensor may be used to characterize the magnitude, the degree of anisotropy, and the orientation of directional diffusion. This review addresses the biological mechanisms, acquisition, and analysis of DTI measurements. The relationships between DTI measures and white matter pathologic features (e.g., ischemia, myelination, axonal damage, inflammation, and edema) are summarized. Applications of DTI to tissue characterization in
\end{abstract}

neurotherapeutic applications are reviewed. The interpretations of common DTI measures (mean diffusivity, MD; fractional anisotropy, FA; radial diffusivity, $D_{\mathrm{r}}$; and axial diffusivity, $D_{\mathrm{a}}$ ) are discussed. In particular, FA is highly sensitive to microstructural changes, but not very specific to the type of changes (e.g., radial or axial). To maximize the specificity and better characterize the tissue microstructure, future studies should use multiple diffusion tensor measures (e.g., $\mathrm{MD}$ and FA, or $D_{\mathrm{a}}$ and $D_{\mathrm{r}}$ ). Key Words: Diffusion tensor imaging, white matter, diffusivity, MRI, brain, fractional anisotropy

\section{INTRODUCTION}

The broad spectrum of magnetic resonance (MR) contrast mechanisms makes MRI one of the most powerful and flexible imaging tool for diagnosis in the CNS. Measurement of the signal attenuation from water diffusion is one of the most important of these contrast mechanisms. In particular, diffusion tensor imaging (DTI) may be used to map and characterize the three-dimensional diffusion of water as a function of spatial location. ${ }^{1,2}$ The diffusion tensor describes the magnitude, the degree of anisotropy, and the orientation of diffusion anisotropy. Estimates of white matter connectivity patterns in the brain from white matter tractography may be obtained using the diffusion anisotropy and the principal diffusion directions. ${ }^{3-5}$

Many developmental, aging, and pathologic processes of the CNS influence the microstructural composition and architecture of the affected tissues. The diffusion of water within tissues will be altered by changes in the tissue microstructure and organization; consequently, diffusion-weighted (DW) MRI methods, including DTI,

Address correspondence and reprint requests to: Andrew L. Alexander, Ph.D., Waisman Laboratory for Brain Imaging and Behavior, Waisman Center, 1500 Highland Ave., Madison, WI 53705. E-mail: alalexander2@wisc.edu. are potentially powerful probes for characterizing the effects of disease and aging on microstructure. Indeed, the applications of DTI are rapidly increasing because the technique is highly sensitive to changes at the cellular and microstructural level.

Methods for the acquisition and analysis of DTI are rapidly evolving and have greatly improved the accuracy of diffusion tensor measurements in recent years, with further improvements to be expected. New pulse sequences and diffusion tensor encoding schemes are continuously being developed to improve the spatial resolution and accuracy and to decrease artifacts in diffusion tensor measurements. The high dimensionality of the diffusion tensor presents both challenges and novel opportunities for describing, visualizing, and analyzing the measurements.

Here we review the principles, methodology, interpretation, and applications of DTI, with discussion of the strengths and weaknesses of the approach and a summary of current extensions of the technology.

\section{BIOLOGICAL DIFFUSION}

Diffusion is a random transport phenomenon, which describes the transfer of material (e.g., water molecules) from one spatial location to other locations over time. In three dimensions, the Einstein diffusion equation ${ }^{6}$ 


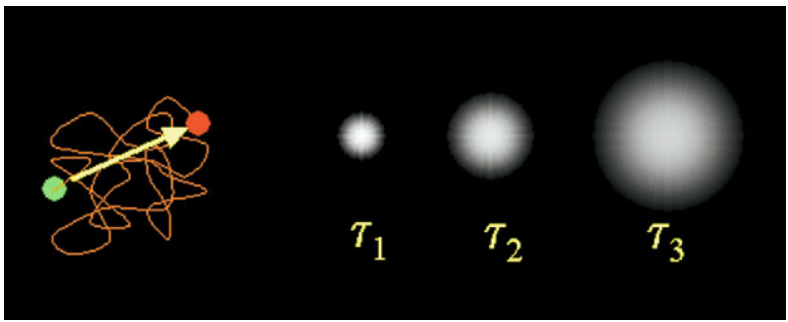

FIG 1. Left: Illustration of the diffusion random walk for a single water molecule from the green location to the red location. The displacement is indicated by the yellow arrow. Right: Diffusion describes the displacement probability with time for a group or ensemble of water molecules. For short diffusion times (e.g., $\left.\Delta t=\tau_{1}\right)$, the predicted spread is compact, but increases with longer diffusion times $\left(\tau_{2}\right.$ and $\left.\tau_{3}\right)$.

$$
D=\frac{<\Delta r^{2}>}{2 n \Delta t}
$$

states that the diffusion coefficient $D$ (in $\mathrm{mm}^{2} / \mathrm{s}$ ) is proportional to the mean squared displacement $\left\langle\Delta r^{2}\right\rangle$ divided by the number of dimensions, $n$, and the diffusion time, $\Delta t$. The diffusion coefficient of pure water at $20^{\circ} \mathrm{C}$ is roughly $2.0 \times 10^{-3} \mathrm{~mm}^{2} / \mathrm{s}$ and increases at higher temperatures. In the absence of boundaries, the molecular water displacement is described by a Gaussian probability density:

$$
P(\Delta r, \Delta t)=\frac{1}{\sqrt{(2 \pi D \Delta t)^{3}}} \exp \left(\frac{-\Delta r^{2}}{4 D \Delta t}\right)
$$

The spread in this distribution increases with the diffusion time, $\Delta t$, as illustrated in Figure 1.

The diffusion of water in biological tissues occurs inside, outside, around, and through cellular structures. Water diffusion is primarily caused by random thermal fluctuations. The behavior is further modulated by the interactions with cellular membranes, and subcellular and organelles. Cellular membranes hinder the diffusion of water, causing water to take more tortuous paths, thereby decreasing the mean squared displacement. The diffusion tortuosity and corresponding apparent diffusivity may be increased by either cellular swelling or increased cellular density. Conversely, necrosis, which results in a breakdown of cellular membranes, decreases tortuosity and increases the apparent diffusivity. Intracellular water tends to be more restricted (as opposed to hindered) by cellular membranes. Restricted diffusion also decreases the apparent diffusivity, but plateaus with increasing diffusion time. ${ }^{7}$ Both hindered and restricted diffusion reduce the apparent diffusivity of water.

In fibrous tissues, including white matter, water diffusion is relatively unimpeded in the direction parallel to the fiber orientation. Conversely, water diffusion is highly restricted and hindered in directions perpendicular to the fibers. Thus, the diffusion in fibrous tissues is anisotropic. Early diffusion imaging experiments used measurements of parallel $\left(D_{\|}\right)$and perpendicular $\left(D_{\perp}\right)$ diffusion components to characterize the diffusion anisotropy. 8,9

The application of the diffusion tensor to describe anisotropic diffusion behavior was introduced by Basser et al. ${ }^{1,2}$ In this elegant model, diffusion is described by a multivariate normal distribution:

$$
P(\Delta \vec{r}, \Delta t)=\frac{1}{\sqrt{(4 \pi \Delta t)^{3}|\mathbf{D}|}} \exp \left(\frac{-\Delta \vec{r}^{\mathrm{T}} \mathrm{D}^{-1} \Delta \vec{r}}{4 \Delta t}\right)
$$

where the diffusion tensor is a $3 \times 3$ covariance matrix

$$
\mathbf{D}=\left[\begin{array}{ccc}
D_{x x} & D_{x y} & D_{x z} \\
D_{y x} & D_{y y} & D_{y z} \\
D_{z x} & D_{z y} & D_{z z}
\end{array}\right],
$$

which describes the covariance of diffusion displacements in three dimensions normalized by the diffusion time. The diagonal elements $\left(D_{i i}>0\right)$ are the diffusion variances along the axes $x, y$, and $z$, and the off-diagonal elements are the covariance terms and are symmetric about the diagonal $\left(D_{i j}=D_{j i}\right)$. Diagonalization of the diffusion tensor yields the eigenvalues $\left(\lambda_{1}, \lambda_{2}, \lambda_{3}\right)$ and corresponding eigenvectors $\left(\epsilon_{1}, \epsilon_{2}, \epsilon_{3}\right)$ of the diffusion tensor, which describe the directions and apparent diffusivities along the axes of principal diffusion.

The diffusion tensor may be visualized as an ellipsoid, with the eigenvectors defining the directions of the principal axes and the ellipsoidal radii defined by the eigenvalues (FIG. 2). Diffusion is considered isotropic when the eigenvalues are nearly equal (e.g., $\lambda_{1} \approx \lambda_{2} \approx \lambda_{3}$ ). Conversely, the diffusion tensor is anisotropic when the eigenvalues are significantly different in magnitude (e.g., $\left.\lambda_{1}>\lambda_{2}>\lambda_{3}\right)$. The eigenvalue magnitudes may be affected by changes in local tissue microstructure with many types of tissue injury, disease, or normal physiological changes (e.g., aging). Thus, the diffusion tensor is a sensitive probe for characterizing both normal and abnormal tissue microstructure.

In the CNS, water diffusion is usually more anisotro-

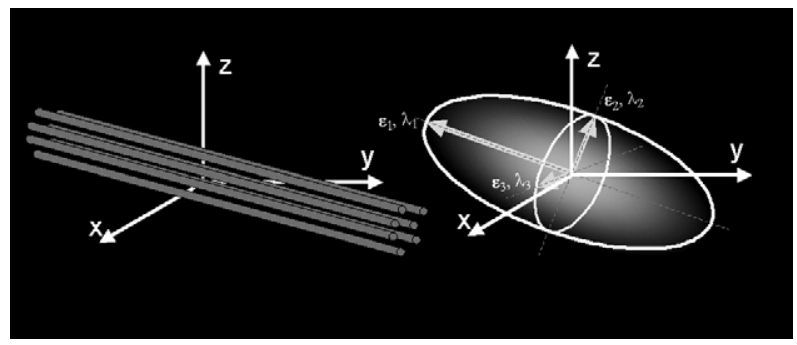

FIG 2. Schematic representation of diffusion displacement distributions for the diffusion tensor. Ellipsoids represent diffusion displacements. The diffusion is highly anisotropic in fibrous tissues such as white matter, and the direction of greatest diffusivity is generally assumed to be parallel to the local direction of the white matter. 


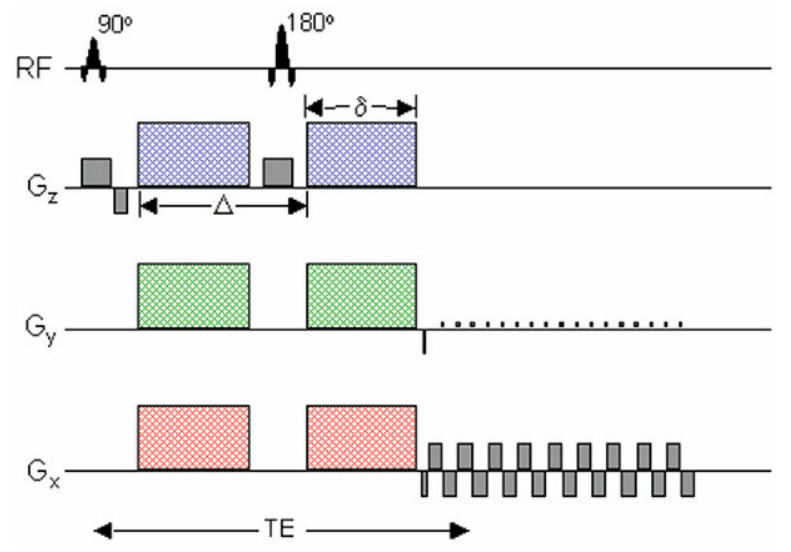

FIG 3. Schematic of a diffusion weighted echo-planar imaging (DW EPI) pulse sequence. A spin echo is used to achieve diffusion weighting from the gradient pulse pairs (colored). The imaging gradients are shown in gray. Diffusion weighting gradients can be applied in any arbitrary direction using combinations of $G_{x}(r e d), G_{y}$ (green), and $G_{z}$ (blue). RF, radiofrequency.

pic in white matter regions and isotropic in both gray matter and cerebrospinal fluid (CSF). The major diffusion eigenvector $\left(\epsilon_{1}\right.$, direction of greatest diffusivity) is assumed to be parallel to the tract orientation in regions of homogeneous white matter. This directional relationship is the basis for estimating the trajectories of white matter pathways with tractography algorithms.

\section{DIFFUSION-WEIGHTED IMAGE ACQUISITION}

The most common diffusion-weighted imaging (DWI) approach is the pulsed-gradient spin echo pulse sequence with a single-shot, echo-planar imaging (EPI) readout (FIG. 3). The simplest configuration of this pulse sequence uses a pair of large-gradient pulses placed on both sides of the $180^{\circ}$ refocusing pulse. The first gradient pulse dephases the magnetization across the sample (or voxel, in imaging); the second pulse rephases the magnetization.

For stationary (nondiffusing) molecules, the phases induced by both gradient pulses will completely cancel, the magnetization will be maximally coherent, and there will be no signal attenuation from diffusion. In the case of coherent flow in the direction of the applied gradient, the bulk motion will cause the signal phase to change by different amounts for each pulse; thus, there will be a net phase difference, which is proportional to the displacement, the area of the diffusion gradient pulses defined by the amplitude, $G$, and the duration, $\delta$, and the spacing between the pulses, $\Delta$.

The displacements of diffusing water are described by a distribution [Eq. (3)]. In the presence of diffusion gradients, therefore, water molecules will accumulate different phases. MRI signals are proportional to the sum of magnetization components from all water molecules in a voxel. Thus, the phase dispersion from diffusion will cause signal attenuation. For simple isotropic Gaussian diffusion, the signal attenuation for the diffusion gradient pulses in Figure 3 is described by

$$
S=S_{o} e^{-b D}
$$

where $S$ is the DW signal, $S_{\mathrm{o}}$ is the signal without any DW gradients (but otherwise identical imaging parameters), $D$ is the apparent diffusion coefficient, and $b$ is the diffusion-weighting described by the properties of the pulse pair:

$$
b(\gamma G \delta)^{2}[\Delta-(\delta / 3)]
$$

where $\gamma$ is the gyromagnetic ratio.

The large diffusion-weighting gradients also make DW MRI extremely sensitive to subject motion. ${ }^{10}$ Even very small amounts of motion can lead to phase and amplitude modulations in the acquired data and significant ghosting artifacts in the reconstructed images. The most widely used DWI method is single-shot EPI, ${ }^{11,12}$ because it is fast, efficient and insensitive to small motion and is readily available on most clinical MRI scanners. A schematic of a DW-EPI pulse sequence is shown in Figure 3. With EPI, the image data for a single slice may be collected in $\leq 100$ $\mathrm{ms}$, effectively freezing any head motion. The fast acquisition speed of EPI makes it highly efficient, which is important for maximizing the image signal-to-noise ratio (SNR) and the accuracy of the diffusion measurements.

Although single-shot EPI is the most common acquisition method for DWI, the disadvantages can be significant. First, both magnetic field inhomogeneities ${ }^{13}$ and eddy currents ${ }^{14}$ can warp the image data, thereby compromising the spatial fidelity. The distortions from diffusion-gradient eddy currents cause misalignments in the DW images, which can lead to subsequent errors in the calculated diffusion maps. These distortions can be described using a combination of translation, scaling, and shear in the phase-encoding direction. ${ }^{14,15}$ To a certain degree, the eddy currents may be reduced using bipolar diffusion-weighting schemes. ${ }^{16,17}$ The distortions may also be retrospectively corrected using image registration methods. ${ }^{14,18,19}$

The image distortion from magnetic field inhomogeneities causes the anatomy of the brain to appear warped nonlinearly in the phase-encoding direction. These effects can be particularly severe in regions near bone-airbrain interfaces, such as the base of the skull, prefrontal areas, and the inferior temporal lobe near the auditory canal. The degree of this distortion is related to the local field variations, the echo spacing or readout bandwidth of the sampling in the phase-encoding direction, the direction of phase encoding, and the field of view. These image distortions are proportional to the magnetic field strength; at $3 \mathrm{~T}$, the distortions can be quite severe. 
Unfortunately, almost none of the published studies of DW-EPI use any form of distortion correction. EPI image distortion can be corrected by measuring a map of the magnetic field strength and correcting the images retrospectively. ${ }^{13}$ Field maps are estimated by measuring the phase difference between gradient echo image data at two different echo times. The acquisition of field map data covering the whole brain can easily be performed in 2-3 minutes. Field maps have been used to correct the EPI distortion in DTI data, which improves the correspondence to non-EPI anatomical image data. This is important for applications such as intraoperative surgical guidance and white matter tractography. Image distortions may also be decreased by a factor of $2-3$ by using parallel imaging methods such as SENSE. ${ }^{20-22}$ Note that, even though the level of distortion is decreased with parallel imaging, it is not completely eliminated, and correction methods are still needed to improve geometric fidelity of diffusion imaging.

The spatial resolution of two-dimensional (2D) EPI pulse sequences also tends to be limited. At $1.5 \mathrm{~T}$, it is possible to acquire $2.5-\mathrm{mm}$ isotropic voxels over the entire brain in roughly 15 minutes. $^{23}$ Smaller voxel dimensions may be achieved using either more sensitive radiofrequency coils or by going to higher field strengths. A recent study by Alexander et al. ${ }^{21}$ demonstrated that the variance in DTI measures (FA and mean diffusivity $[\mathrm{MD}]$ ) was reduced by roughly a factor of 2 at $3 \mathrm{~T}$ relative to $1.5 \mathrm{~T}$, although the distortions were greater at $3 \mathrm{~T}$.

\section{DIFFUSION TENSOR ENCODING}

A minimum of six noncollinear diffusion encoding directions are required to measure the full diffusion tensor. ${ }^{24,25}$ A wide variety of diffusion tensor encoding strategies have been proposed, with six or more encoding directions. ${ }^{25-29}$ The selection of tensor encoding directions is critical for accurate and unbiased assessment of diffusion tensor measures. Hasan et al. ${ }^{29}$ performed a comprehensive comparison of various heuristic, numerically optimized, and natural polyhedra encoding sets. This study demonstrated that encoding sets with uniform angular sampling yield the most accurate diffusion tensor estimates. Recent studies have provided mounting evidence that more diffusion encoding directions causes the measurement errors to be independent of the tensor orientation. ${ }^{30}$

\section{PROTOCOL CONSIDERATIONS}

Multiple considerations are involved in the choice of a diffusion tensor protocol. The protocol choice is moderately complicated by the wide spectrum of pulse sequence parameters to be configured. As already mentioned, the DW spin-echo, single-shot EPI is the most common pulse sequence for DTI. The optimum diffusion weighting (i.e., the $b$-value) for the brain is roughly between 700 and $1300 \mathrm{~s} / \mathrm{mm}^{2}$, with a $b$-value of $1000 \mathrm{~s} / \mathrm{mm}^{2}$ being most common. The selection of the number of encoding directions depends on the availability of encoding direction sets, the desired scan time, and the maximum number of images that can be obtained in a series.

Measurements of diffusion anisotropy tend to be sensitive to image noise, which can also lead to biases in the anisotropy estimates. ${ }^{31}$ The accuracy of DTI measures may be improved by increasing either the number of encoding directions or the number of averages. Unfortunately, this increases the scan time for DTI data collection. The image SNR can also obviously be improved by using larger voxels, although this will increase partial volume averaging of tissues, which can lead to errors in the fits to the diffusion tensor model. ${ }^{32}$

The specific image acquisition settings for a protocol will depend on the application. For many routine clinical applications (brain screening, stroke, brain tumors), a fairly coarse spatial resolution can be used with a small number of encoding directions. For applications requiring accurate quantification, however, such as quantifying changes in multiple sclerosis (MS) plaques, comparing DTI measures between different neurological or psychiatric groups, quantifying DTI measures in very small white matter tracts, or estimating white matter trajectories with white matter tractography, high spatial resolution is much more important and a large number of diffusion encoding directions or averaging is desirable. High-quality DTI data with whole-brain coverage, $2.5-\mathrm{mm}$ isotropic resolution and 64 diffusion encoding directions may be obtained in $\sim 15$ minutes on clinical 1.5-T scanners. ${ }^{23}$ Similar DTI data quality may be achieved in almost one quarter of the time at $3.0 \mathrm{~T}$-but the image distortions are roughly double. ${ }^{21}$

\section{DIFFUSION TENSOR IMAGE PROCESSING}

Maps of DTI measures are estimated from the raw DW images. As already discussed, the images may be distorted and misregistered from a combination of eddy currents, subject motion, and magnetic field inhomogeneities. Ideally, these distortions and sources of misregistration would be corrected before calculating any subsequent quantitative diffusion maps. Methods for image correction and coregistration have already been touched on briefly. 


\section{CALCULATION OF APPARENT DIFFUSIVITIES AND DIFFUSION TENSOR ELEMENT MAPS (DECODING)}

The first step in the calculation of the diffusivities tensor is to estimate the apparent diffusivity maps, $D_{i, \text { app }}$, for each encoding direction. Equation (5) has to be adjusted to describe the signal attenuation for anisotropic diffusion with the diffusion tensor:

$$
S_{i}=S_{o} \exp ^{-b \hat{g}_{\mathrm{i}}^{\mathrm{T} D \hat{g}_{\mathrm{i}}}}=S_{o} e^{-b_{i} D_{i, \mathrm{app}}}
$$

where $S_{i}$ is the DW signal, the index $i$ corresponds to a unique encoding direction, $\hat{\mathbf{g}}_{i}$ is the unit vector describing the DW encoding direction, and $b_{i}$ is the amount of diffusion weighting in Equation (6). In the case of single diffusion weighting ( $b$-value) and an image with very little or no diffusion weighting $\left(S_{\mathrm{o}}\right)$, the apparent diffusivity maps are estimated via

$$
D_{i, \text { app }}=\frac{\ln \left(S_{i}\right)-\ln \left(S_{o}\right)}{b_{i}}
$$

Subsequently, the six independent elements of the diffusion tensor $\left(\mathrm{D}_{x x}, \mathrm{D}_{y y}, \mathrm{D}_{z z}, \mathrm{D}_{x y}=\mathrm{D}_{y x}, \mathrm{D}_{x z}=\mathrm{D}_{z x}\right.$, and $\mathrm{D}_{y z}=\mathrm{D}_{z y}$ ) may be estimated from the apparent diffusivities using multiple linear least squares methods ${ }^{1,29}$ or nonlinear modeling. ${ }^{33}$

\section{DIFFUSION TENSOR IMAGE MEASURES}

The display, meaningful measurement, and interpretation of $3 \mathrm{D}$ image data with a $3 \times 3$ diffusion matrix at each voxel is a challenging or impossible task without simplification of the data. Consequently, it is desirable to distill the image information into simpler scalar maps. The two most common measures are the trace and anisotropy of the diffusion tensor. The trace of the tensor (Tr), or sum of the diagonal elements of $\mathbf{D}$, is a measure of the magnitude of diffusion and is rotationally invariant. The MD (also called the apparent diffusion coeffcient, or ADC) has been used in many published studies and is simply the trace divided by $3(\mathrm{MD}=\mathrm{Tr} / 3)$, which is equivalent to the average of the eigenvalues.

The degree to which the diffusivities are a function of the DW encoding direction is represented by measures of diffusion anisotropy. Many measures of anisotropy have been described, most of which are rotationally invariant. ${ }^{34-38}$ Currently, the most widely used invariant measure of anisotropy is the fractional anisotropy (FA) described originally by Basser and Pierpaoli ${ }^{33}$ :

$$
\mathrm{FA}=\sqrt{\frac{\left(\lambda_{1}-\mathrm{MD}\right)^{2}+\left(\lambda_{2}-\mathrm{MD}\right)^{2}+\left(\lambda_{3}-\mathrm{MD}\right)^{2}}{2\left(\lambda_{1}^{2}+\lambda_{2}^{2}+\lambda_{3}^{2}\right)}}
$$

Note that the FA does not describe the full tensor shape or distribution. This is because different eigen- value combinations can generate the same values of FA. ${ }^{39}$ Although FA is likely to be adequate for many applications and appears to be quite sensitive to a broad spectrum of pathological conditions, the full tensor shape cannot be simply described using a single scalar measure. ${ }^{39}$ The tensor shape can, however, be described completely using a combination of spherical, linear, and planar shape measures. ${ }^{36,39}$

Several recent studies have also suggested that the eigenvalue amplitudes or combinations of the eigenvalues-e.g., the radial diffusivity, $D_{\mathrm{r}}=\left(\lambda_{2}+\lambda_{3}\right) / 2-$ demonstrate more specific relationships to white matter pathology. For example, the $D_{\mathrm{r}}$ appears to be modulated by myelin in white matter, whereas the axial diffusivity $\left(D_{\mathrm{a}}=\lambda_{1}\right)$ is more specific to axonal degeneration. ${ }^{40}$ Consequently, it is important to consider alternative quantitative methods when trying to interpret DTI measurements.

Another important measure is the tensor orientation described by the major eigenvector direction. For diffusion tensors with high anisotropy, the major eigenvector direction is generally assumed to be parallel to the direction of white matter tract, which is often represented using a red-green-blue (RGB) color map to indicate the eigenvector orientations. ${ }^{41,42}$

The local eigenvector orientations can be used to identify and parcellate specific WM tracts; thus, DT-MRI has an excellent potential for applications that require high anatomical specificity. The ability to identify specific white matter tracts on the eigenvector color maps has proven useful for mapping white matter anatomy relative to lesions for preoperative planning ${ }^{43}$ and postoperative follow-up. ${ }^{44}$ Statistical methods have recently been developed for quantifying the distributions of tensor orientation in specific brain regions. ${ }^{45}$ Maps of the MD, FA, major eigenvector direction, and eigenvalues are shown as examples in Figure 4.

Histograms of FA, MD, $D_{\mathrm{a}}$, and $D_{\mathrm{r}}$ in healthy gray matter (GM), white matter (WM), and CSF are plotted in Figure 5. The histograms demonstrate considerable overlap in the measurement distributions of the major tissue groups. The FA measurements in WM have a very broad distribution with considerable skew and kurtosis. (FA ranges between 0 and 1.) The other measures in GM and WM appear more normally distributed (not tested), with the biggest difference between tissue types in the $D_{\mathrm{r}}$ measure.

\section{EFFECTS OF NOISE ON DT-MRI}

The measures derived from the diffusion tensor are moderately sensitive to noise, primarily due to use of multiple noisy images for the estimation. The effects of Gaussian random noise on the tensor trace and anisotropy measures have been studied using Monte Carlo 


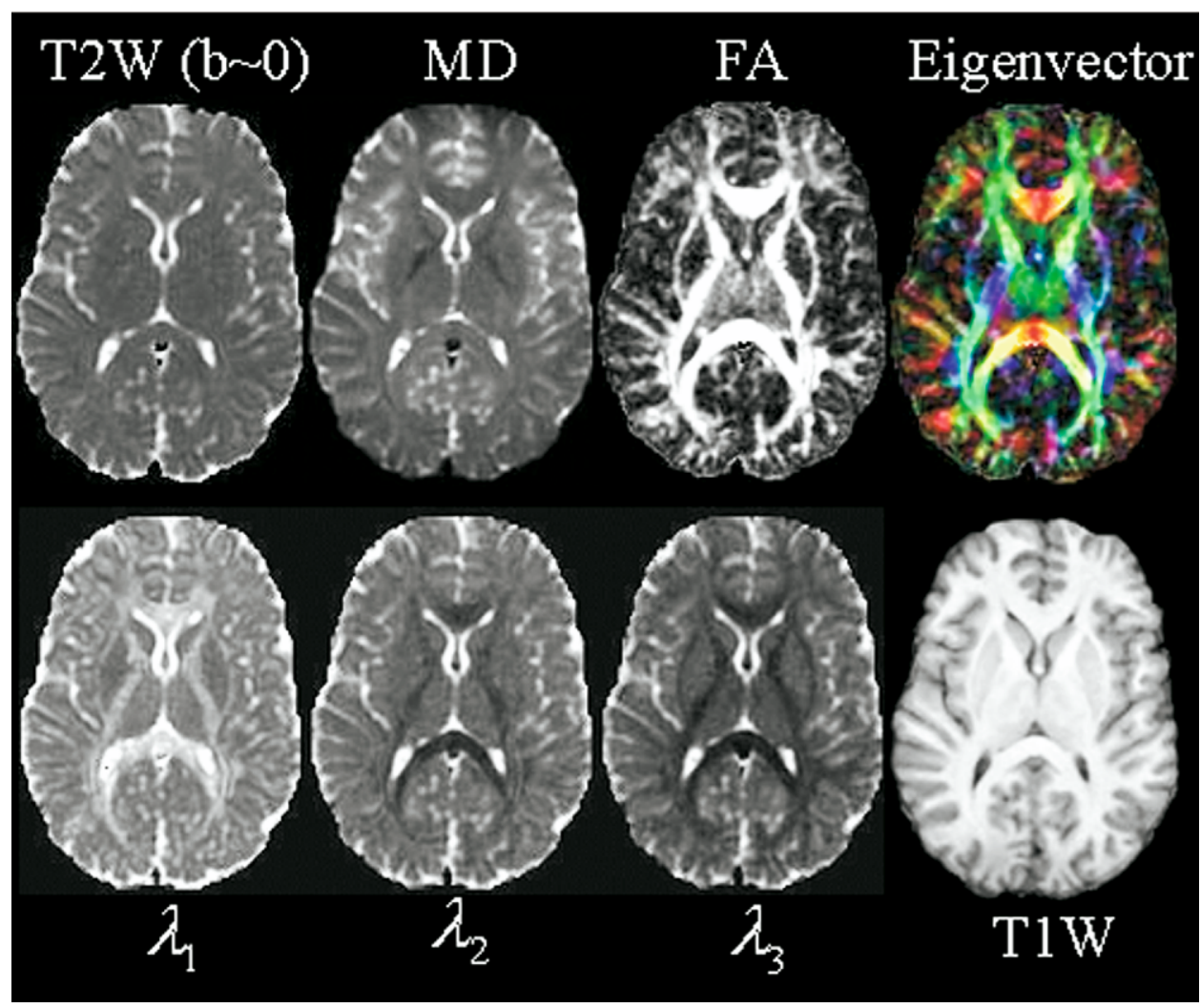

FIG 4. Quantitative maps from a diffusion tensor imaging (DTI) experiment. The images show (left to right, top row first) the $T_{2}$-weighted $(\mathrm{T} 2 \mathrm{~W})$ reference image (i.e., $b=0$ ) from DTI data; the mean diffusivity (MD; note similar contrast to T2W image with CSF appearing hyperintense); fractional anisotropy (FA; hyperintense in white matter); the major eigenvector direction indicated by color (red: right-left; green: anterior-posterior; blue: superior-inferior) weighted by the FA (note that specific tract groups can be readily identified); the major, medium, and minor eigenvectors $\left(\lambda_{1}, \lambda_{2}\right.$, and $\lambda_{3}$, respectively); and, last, a conventional $\mathrm{T}_{1}$-weighted image from three-dimensional MP-RAGE (magnetization-prepared rapid gradient echo) at the same anatomical location.

noise simulation studies. ${ }^{31}$ The noise and eigenvalue sorting process tend to cause overestimation of the major eigenvalue and underestimation of the minor eigenvalue and thus an increase in the uncertainty of all the eigenvalues. In addition, the eigenvector orientations tend to be highly sensitive to noise. ${ }^{46}$ Consequently, for applications where quantitative accuracy are important, it is critical to acquire DTI data with adequate SNR.

\section{WHITE MATTER TRACTOGRAPHY}

The orientation of the diffusion tensor major eigenvector is generally assumed to be parallel to the local white matter fascicles. These directional patterns may be simply visualized using the color maps representing the major eigenvector direction, similar to the example in Figure 4 . These color maps are very useful for surveying the organization of white matter in the brain and for identifying major white matter tracts in 2D sections. ${ }^{42,43}$

Another approach for appreciating the white matter connection patterns in 3D is using white matter tractography, which follows coherent spatial patterns in the major eigenvectors of the diffusion tensor field. ${ }^{3-5}$ White matter patterns are estimated by starting at a specified location (called the seed point), estimating the direction of propagation (often defined as the major eigenvector), and moving a small distance in that direction (called tract integration). The tract direction is then re-evaluated and another small step is taken, and so on, until the tract is terminated. Tracts may be constrained by using one or more regions of interest. ${ }^{47,48}$ Most algorithms use the major eigenvector to estimate the tangent of the trajectory for a white matter fiber bundle, although tracing methods based on the full diffusion tensor field have also been developed. ${ }^{36,49}$

With these methods, tractography algorithms are capable of generating anatomically plausible estimates of white matter trajectories in the human brain. Tractography methods have been used to generate anatomically plausible tract reconstructions of major projection pathways (e.g., corticospinal tract, corona radiata), commissural pathways (e.g., corpus callosum, anterior commissure), and association pathways (e.g., arcuate fasciculus, inferior longitudinal fasciculus, uncinate fasciculus, etc.). ${ }^{47,48,50-52}$ The primary applications of tractography to date have been the visualization of WM trajectories in 3D (particularly in relation to brain pathology) and seg- 


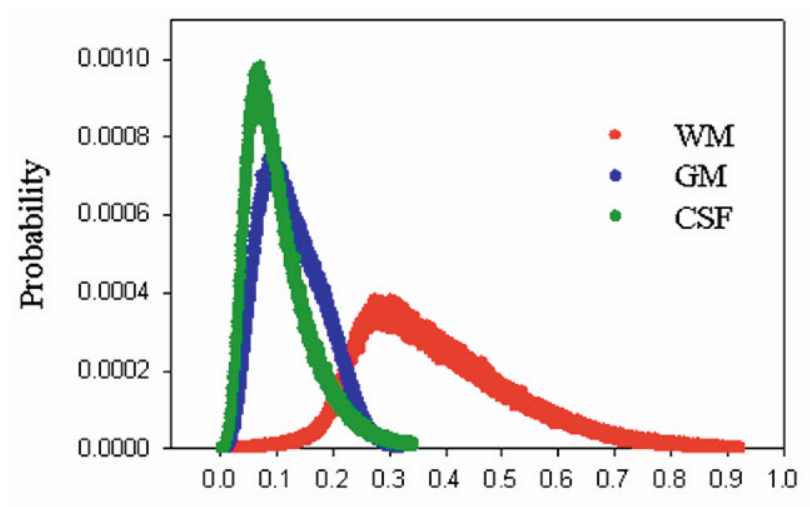

FA

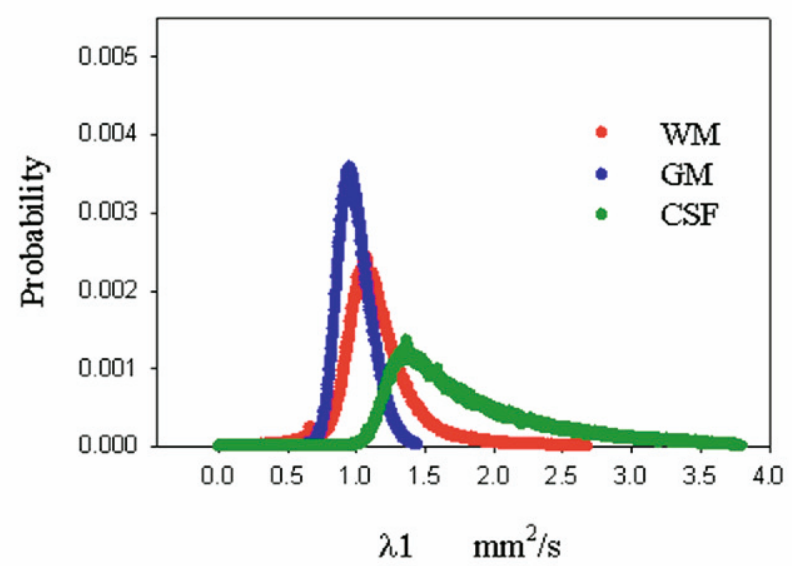

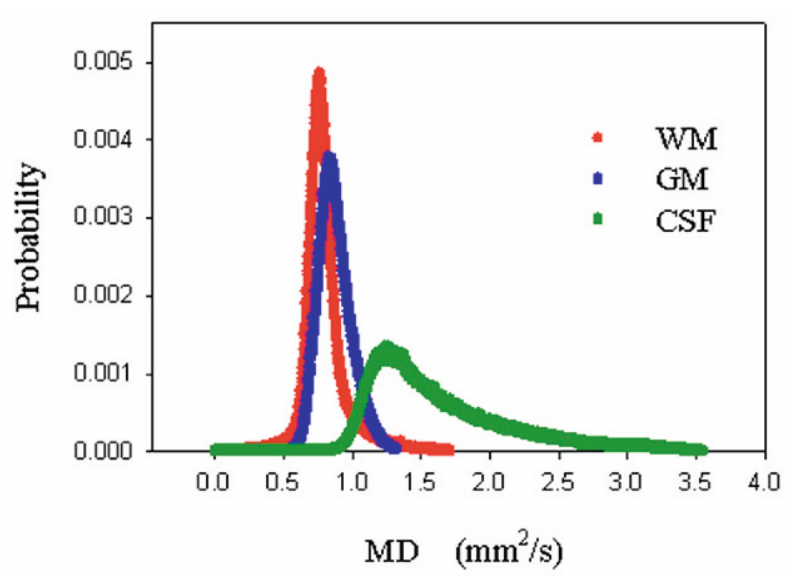

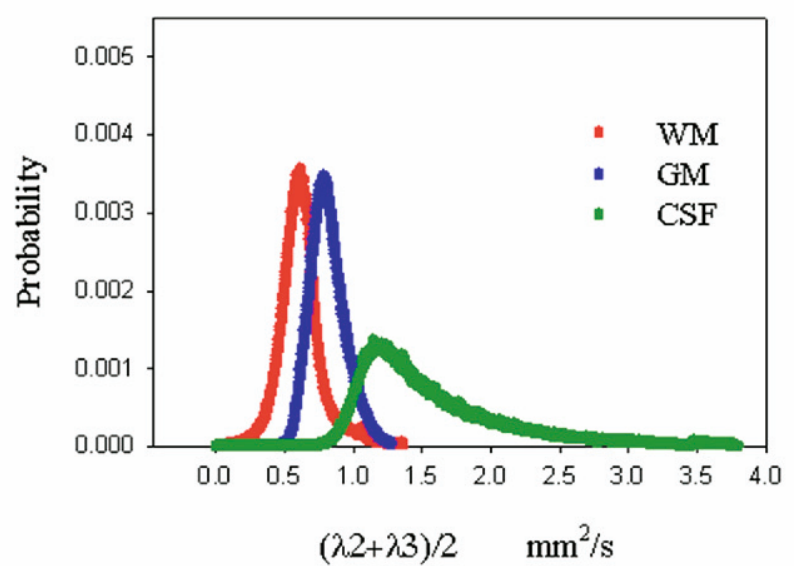

FIG 5. Histogram distribution plots of FA, MD, axial diffusivity $D_{\mathrm{a}}\left(=\lambda_{1}\right)$, and radial diffusivity $D_{\mathrm{r}}\left[=\left(\lambda_{2}+\lambda_{3}\right) / 2\right]$ for whole-brain gray matter (GM; blue), white matter (WM; red), and CSF (green).

mentation of specific brain regions. An tractogram of the corona radiata (projection fibers from brainstem to cortex) in a healthy subject is shown as an example in Figure 6. Tractography-based segmentation of major white matter pathways is depicted in Figure 7.

Despite the promising results obtained in these studies, tractography has several limitations. Estimates of the eigenvector directions, and hence the local tract directions, are sensitive to thermal noise, physiologic fluctuations, and image artifacts. Algorithms based on the major eigenvector cannot resolve regions of crossing white matter pathways. New diffusion imaging methods, such as QBI (q ball imaging), ${ }^{53}$ HARDI (high angular diffusion imaging), ${ }^{54,55}$ CHARMED (combined hindered and restricted diffusion), ${ }^{56}$ and DSI (diffusion spectrum imaging $)^{57}$, may be better able to resolve the intersections of crossing white matter regions more accurately. These methods require higher diffusion weighting (typically 3000-15,000 s/ $\mathrm{mm}^{2}$ ), however, and take much more time in acquisition. Tractography results with these methods are promising. ${ }^{58}$

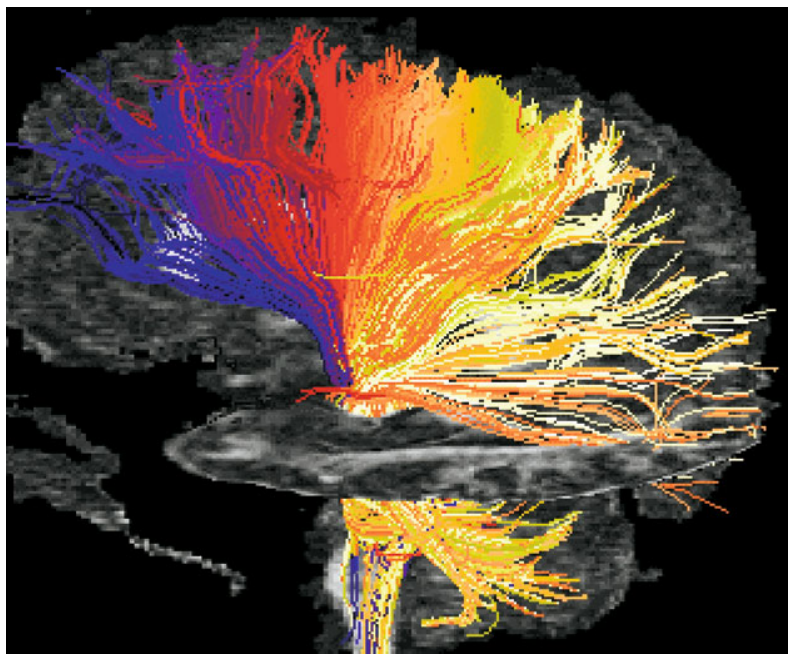

FIG 6. Tractography image of corona radiata in healthy subject. Color was used to label tract termination at cortex (the color sequence blue to yellow corresponds to anterior to posterior location). 


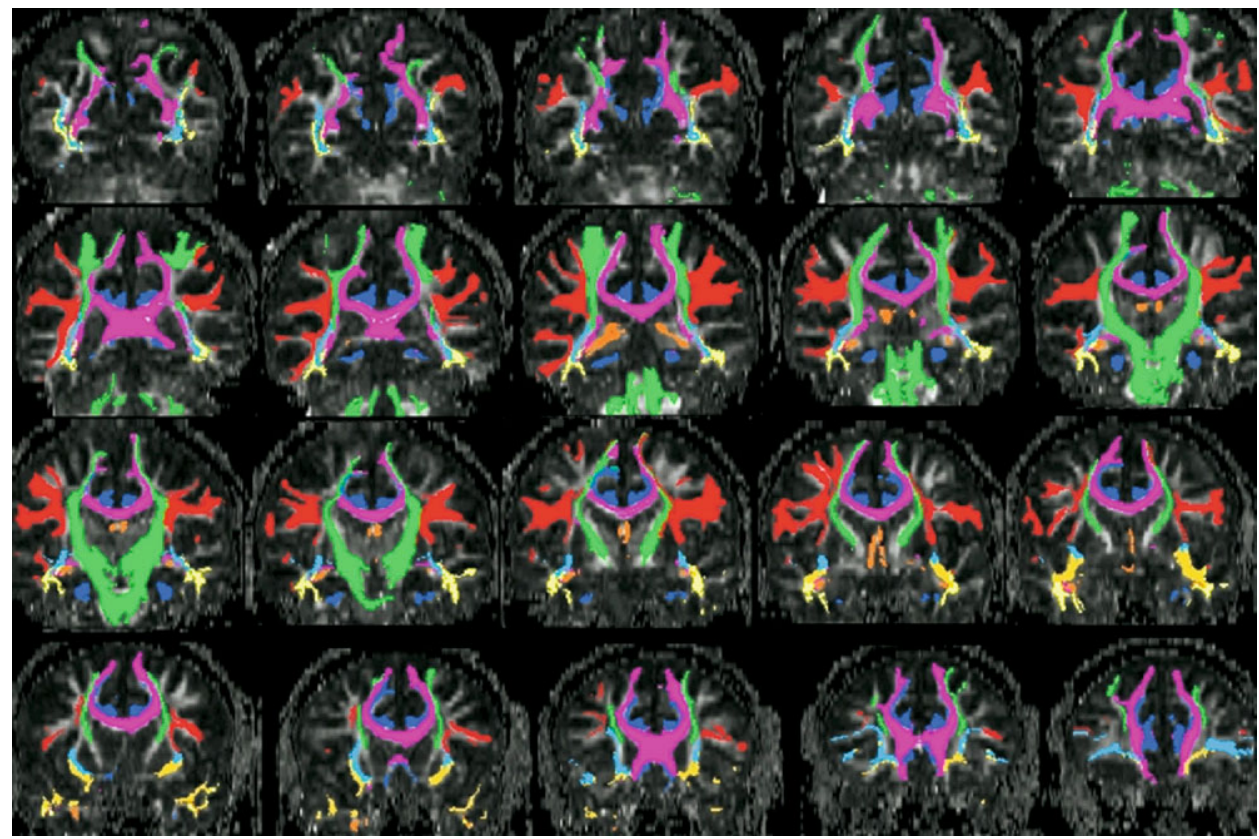

FIG. 7. WM parcellation of major tracts for one subject using tractography. DTI data were collected with 2.3-mm isotropic resolution. The tracts shown include the superior longitudinal fasciculus (red); corpus callosum (purple); inferior occipital fasciculus (light blue); inferior longitudinal fasciculus (yellow); uncinate fasciculus (orange); fornix/stria terminalis (dark orange); and corona radiata (green).

\section{INTERPRETATION OF DTI MEASURES}

DTI has been used in a broad spectrum of applications. The primary reason is that water diffusion in tissues is highly sensitive to differences in the microstructural architecture of cellular membranes. Increases in the average spacing between membrane layers will increase the apparent diffusivity, whereas smaller spaces will lead to lower apparent diffusivities. This sensitivity makes DTI a powerful method for detecting microscopic differences in tissue properties.

Nonetheless, the interpretation of changes in the measured diffusion tensor is complex and should be performed with care. Many published research studies have focused primarily on the diffusion anisotropy (usually the FA measure), which may not be enough to characterize the tissue changes. For example, white matter (WM) neuropathology often causes the anisotropy to decrease, which may result from increased radial (perpendicular) diffusivity, reduced axial (parallel) diffusivity, or both. Measurements of the MD or trace may help to better understand how the diffusion tensor is changing. Alternatively, more recent studies have started to examine measurements of either the eigenvalues or the radial and axial diffusivities directly, to provide more specific information about the diffusion tensor. ${ }^{40,59}$

Interpretation is further complicated by the sensitivity of the diffusion tensor, and the anisotropy in particular, to a broad spectrum of other factors, including image noise (both thermal and physiologic), ${ }^{31,46,60}$ artifacts (e.g., misregistration of DW images from eddy currents or head motion), partial volume averaging between tis- sues in large voxels (e.g., signal mixing of gray matter, WM and CSF), ${ }^{32}$ and regions of crossing WM tracts. ${ }^{32,61}$ The last confound is unfortunately unavoidable, because many areas of the brain have considerable areas of fiber crossing (e.g., the centrum semiovale, uncinate fasciculi, and transpontine fibers), which have corresponding low FA. Consequently, changes in the angle and relative volume fractions of crossing WM fiber groups within a voxel can result in significant anisotropy changes without any white matter abnormalities.

Within healthy WM, FA can range between values of 0.1 to almost 1.0 (peaking at $\sim 0.3$ ) (FIG. 5), and much of this variation is caused by crossing WM fibers. The variability in FA can be reduced considerably by focusing on white matter tracts in specific anatomic regions, particularly with fewer white matter crossings: for example, homogeneous areas with a single fiber population, such as the pyramidal tracts and the corpus callosum (before intersection within the centrum semiovale).

In the absence of other information, FA is thus a highly sensitive but fairly nonspecific biomarker of neuropathology and microstructural architecture. This combination imposes challenges on the interpretation of DTI measurements for diagnostic and therapeutic applications. Many studies imply that FA is a marker of socalled white matter integrity-but these claims are far from definitive.

Despite these limitations, however, DTI is certainly a sensitive marker of neuropathology. Hundreds of research studies have observed reduced FA in a broad spectrum of diseases, with increases rarely reported. Fur- 
thermore, if the neuropathologic basis for a specific disease is understood, then the results may be interpreted with greater specificity. For example, demyelination might cause the radial diffusivity to increase, with minimal influence on the axial diffusivity. Increased tissue water in edema will increase the MD, whereas cell proliferation in neoplasia may decrease the MD. Conversely, in complex diseases such as MS, brain regions may experience an unpredictable combination of demyelination, axon loss, gliosis, and inflammation, which could result in competing influences on the diffusion tensor.

Use in combination with other imaging measures (e.g., $\mathrm{T}_{1}, \mathrm{~T}_{2}$, magnetization transfer, perfusion, fast/slow diffusion, spectroscopy) may help to improve the specificity of DTI in complex diseases. In relatively homogeneous (single-fiber), well-characterized white matter tissues, the diffusion tensor appears to have various relationships to neuropathology. Several are discussed here: ischemic stroke, demyelination and dysmyelination, inflammation, edema, and neoplasia.

\section{Ischemic stroke}

The clinical diagnosis and characterization of acute ischemic lesions in the CNS is by far the most common clinical application of DWI. In the acute phase of brain ischemia, the MD (or trace) significantly decreases in the lesion. ${ }^{62}$ After several days $(\sim 5-7)$, the MD starts to renormalize (appear normal). In contrast, chronic ischemic lesions ( $>2$ weeks) typically demonstrate significantly increased MD as encephalomalacia ensues. Recent studies have also shown that the FA appears to increase in acute lesions and decrease below baseline levels in the chronic phase. ${ }^{63-65}$ Investigations of specific eigenvalues appear to show decreases in the first and second (largest and medium) eigenvalues during acute ischemia, relative to the contralateral hemisphere. ${ }^{64}$

\section{Demyelination and dysmyelination}

Early studies demonstrated that the parallel organization of white matter fiber bundles is the basis for diffusion anisotropy, whereas myelin appears to modulate the amount of anisotropy. ${ }^{66}$ Nearly all studies of myelination with normal brain development ${ }^{67}$ or demyelination with disease-related processes have found less diffusion anisotropy when axons are less myelinated.

During early brain development, all three eigenvalues appear to decrease with maturation, although the decline in diffusivity is more substantial in the radial diffusion orientations (medium and minor eigenvalues), consistent with the development of myelination ${ }^{68}$ (although many other processes are also occurring during brain maturation). Recently, Song et al. ${ }^{40}$ examined the radial and axial diffusivities of white matter in a mouse model of dysmyelination and found that the absence of myelin appeared to increase the radial diffusivity, but did not significantly affect the axial diffusivity. Subsequent studies have confirmed increased radial diffusivity in models of dysmyelination ${ }^{69}$ and demyelination ${ }^{70,71}$; a few studies, however, observed decreased axial diffusivity with dysmyelination or demyelination-although these differences were less significant. ${ }^{69,71}$

Axonal damage and loss may also have modulatory effects on these measures, and recent studies ${ }^{71,72}$ have suggested that the axial diffusivity may be a more specific marker of axonal damage. Note that these studies focused on homogeneous parallel WM regions, which are not significantly confounded by crossing WM fibers. Regardless of the specific mechanisms, in comparison to the diffusion anisotropy, the axial and radial diffusivities (or diffusion eigenvalues) provide more specific information about diffusion tensor changes or differences. Recently, more studies have started to examine the axial and radial diffusivities using DTI. Increased radial diffusivities have been observed in high anisotropy WM of patients with relapsing-remitting $\mathrm{MS},{ }^{73}$ in periventricular frontal WM in early Alzheimer's disease, ${ }^{74}$ in periventricular WM of patients with hydrocephalus, ${ }^{75}$ in remaining corpus callosum WM tracts post corpus callosotomy,${ }^{76}$ in extratemporal $\mathrm{WM}$ in patients with temporal lobe epilepsy, ${ }^{77}$ in the genu of the corpus callosum of cocaine addicts, ${ }^{78}$ and in the corpus callosum of subjects with autism. ${ }^{59}$ In many of these cases, myelination may play a significant modulatory role in the radial diffusivity.

\section{Inflammation}

To date, very little work has been done to characterize the relationships between DTI measures and inflammation. Generally, the increased tissue water engendered by inflammation also increases the MD, which will result in a decrease in the diffusion anisotropy. In one study, the MD appeared to be highly elevated in acute MS lesions, whereas chronic and subacute lesions exhibited less elevated mean diffusivities. ${ }^{79}$ In a separate study, Werring et al. ${ }^{80}$ found that destructive $\mathrm{T}_{1}$ hypointense lesions showed the greatest elevation in MD and that contrastenhancing lesions (inflammatory) showed the largest decrease in FA.

\section{Edema}

The effects of edema on DTI measurements are similar to that of inflammation, as one would expect: the MD is increased and the anisotropy is decreased. This pattern of DTI measures is a general hallmark of many disease and injury processes, which, as we have discussed, limits the specificity of DTI measurements. One observation of note is that, although the anisotropy is reduced, the directional patterns of the affected WM tracts appear unaltered, whereas glioma infiltration may cause alterations in the WM fiber orientations. ${ }^{45,81}$ 


\section{Neoplasia}

Possibly the second most common clinical application of DTI is for the characterization of white matter in patients with brain tumors. Much of this work focuses on using DTI maps and tractography to help localize WM fiber tracts that are important for such critical functions as motion, language, and vision. ${ }^{43,44,52}$ Armed with this information, the neurosurgeon can plan surgical procedures that will minimize injury to critical tracts, such as the corticospinal tract. ${ }^{82}$

DTI has also been applied to characterize tissues, albeit with limited success. The heterogeneity of brain tumors in the presence of complex environments (e.g., edema, mass effects) and the inherent heterogeneity of diffusion anisotropy in normal white matter reduces the overall specificity of DTI measures. In general, it is assumed that increased cellular densities will decrease the MD and that the MD will be significantly elevated in areas of tissue necrosis.

A study of pediatric tumor patients revealed relationships between MD and both tumor grade and cellularity. ${ }^{83}$ Another study compared MD measurements in lymphomas (with high cellularities) and high-grade astrocytomas, with the finding that the diffusivities were significantly higher in the astrocytomas, whereas the diffusivities in the lymphomas were only slightly or not elevated relative to normal-appearing tissue measured in the contralateral hemisphere. ${ }^{84}$ Beppu et al. ${ }^{85,86}$ reported correlations between FA and cell density and proliferation in both astrocytomas and glioblastomas, with higher FA values corresponding to higher cell densities.

Investigators have also tried to use DTI to differentiate between gliomas and edema, which is challenging with conventional MRI methods. As we have noted, tract orientations appear to be highly affected by infiltrating gliomas, whereas the directions appear to be preserved in regions of edema. Lu et al.$^{87}$ developed a criterion (the Tumor Infiltration Index) based on the relationships between FA and MD to classify neoplasia and peritumoral edema. Other studies using DTI have found no significant differentiation between tumor types and edema. ${ }^{88,89}$ The type of edema may also influence the diffusion properties. In one recent study, peritumoral edema from high-grade tumors (relative to low-grade) appeared to have higher diffusivity, possibly from the destruction of the extracellular tissue matrix. ${ }^{90}$

\section{ROLE OF DTI IN NEUROTHERAPEUTICS}

The high sensitivity but low specificity of DTI measures presents challenges for its diagnostic application in neuropathology. It is, however, a potentially powerful technique for monitoring the response of the brain to therapies. Because subjects serve as their own baseline reference, it is possible to follow subjects longitudinally, to determine how the microstructural properties of the tissue change over time. Furthermore, if the type of injury is well characterized or the therapeutic effects are predictable, the specificity of the measured changes may be greatly increased. Several of the potential applications in the assessment and management of patients with neuropathology are surveyed here.

\section{Predictors of disease course and therapeutic response}

Image-based prognostic indicators of disease course and response to therapy would be extremely valuable. Predictive imaging measures would enable earlier interventions and would be useful for assessing which patients are most likely to respond to specific therapies. Several recent studies have examined DTI measures for predicting disease risk and response factors. Conventional imaging is particularly challenging in newborns and young children. DTI offers unique contrast in WM that may be more sensitive to injury and abnormal development. A study of low birth weight preterm infants found that the FA in the posterior limb of the internal capsule was significantly reduced in the most neurologically abnormal infants. ${ }^{91}$ At the other end of the aging range, DTI has been used to characterize patients at risk for Alzheimer's disease and late-onset depression. In a small pilot study of patients with mild cognitive impairment, the MD of the hippocampus was a better predictor than the hippocampus volume for patients who progressed to Alzheimer's disease. ${ }^{92}$ Diminished FA in white matter regions including anterior cingulate and dorsolateral frontal pathways in patients with late-life depression was found to be a strong predictor of poor response to the antidepressant citalopram. ${ }^{93}$

DTI measures have also been evaluated as prognostic indicators of disease progression and response to therapy in lesion-based neuropathology. Mean diffusivity of normal appearing white matter was found to correlate with cognitive performance (IQ and executive function) measures in patients with ischemic leukoaraiosis. ${ }^{94}$ Similarly in CADASIL (i.e., cerebral autosomal dominant arteriopathy with subcortical infarcts and leukoencephalopathy), a recent study demonstrated that increased average MD from whole-brain histograms was the best predictor of future clinical progression..$^{95}$ Conversely, in the case of ischemic stroke, changes in either the MD or apparent diffusion abnormality in the ischemic tissue during the acute phase were not found to be predictive of the clinical outcome. ${ }^{96}$

In chronic stroke patients, however, FA measurements of the corticospinal tract were found to be correlated with motor functional recovery. ${ }^{97}$ In that study, functional MRI and WM tractography were used to localize the corticospinal tract. This unique ability of DTI to map the trajectories of specific WM tracts is also promising for 
mapping abnormalities to functionally specific anatomy. One recent study used tractography to study the corpus callosum and the corticospinal tracts in relapsing-remitting MS patients. ${ }^{98}$ The MD of the corticospinal tract correlated with the pyramidal Fatigue Severity Scale clinical score, which is related to motor function. The MD of the corpus callosum, however, correlated with the PASAT score (Paced Auditory Serial Addition Test), which characterizes function associated with auditory attention and cognitive processing speed.

\section{Surgical interventions}

DTI has been used widely for mapping WM anatomy prior to surgery. ${ }^{43,52,99}$ This assists the clinical intervention team with localization of critical white matter pathways, to minimize damage to these areas. These pathways may be visualized using either color eigenvector maps (FIG. 4; see also Witwer et al. ${ }^{43}$ ) or tractography. ${ }^{44}$

A recent study by Powell et al. ${ }^{100}$ found that tractography could predict visual field deficits that occurred after resection of the temporal lobe. DTI and tractography have also been implemented in the intraoperative setting to facilitate real-time WM tract mapping, to compensate for shifting tissues during the surgery. ${ }^{101-104}$ These visualization techniques have also been applied after surgical intervention to assess the effects of the surgery on the WM tracts. ${ }^{14,82,99}$

In addition to visualization of the WM tracts, DTI has also been used to study the effects of surgery on WM pathways. A small DTI study of patients with hemispherectomies revealed no significant changes in either the MD or FA in the contralateral corticospinal tract ${ }^{105}$; however, the ipsilateral pontine and cerebral peduncle fiber regions demonstrated increased MD and reduced FA, likely from Wallerian degeneration. Similar Wallerian degeneration changes in the DTI measures were observed in the optic radiations following temporal lobectomy. ${ }^{106}$

Detailed DTI studies of patients following corpus collosotomy found reduced FA following the surgical intervention (the anterior $2 / 3$ of the corpus callosum is bisected). At 1 week after surgery, however, the FA decrease was attributed to increased axial diffusivity (from axonal fragmentation), whereas the FA decrease 2-4 months after the surgery was attributed to increases in the radial diffusivity (consistent with demyelination). ${ }^{76}$

\section{Radiation treatment in neoplasia}

Several studies have demonstrated that radiation therapy decreases the FA of affected WM regions. ${ }^{107-109}$ This decrease in FA appears to be related to the overall radiation dose, and so may be used to assess dose distribution. ${ }^{108}$ The MD and FA measures also appear to be promising for differentiating between recurrent brain tumors and radiation injury in regions of new contrast- enhancing lesions. ${ }^{109}$ Two recent studies have also examined the relationships between DTI changes from radiation and cognitive function as measured by IQ. ${ }^{10,111}$ Both studies reported a correlation between decreased FA and decreased IQ. The Mabbott et al. ${ }^{110}$ study also observed that increased MD in WM correlated with decreased IQ.

\section{Other treatments}

DTI has also been used to follow the response of neuropathology to pharmacological therapies. One example is a longitudinal study of dystonia patients after botulinum toxin treatment. ${ }^{112} \mathrm{FA}$ measurements in the posterior limb of the interior capsule appeared to normalize after 4 weeks of treatment. DTI has also been used to investigate the effects of steroid hormone dexamethasone treatment on peritumoral edema ${ }^{113}$; this study demonstrated a reduction in the MD of the edematous region with no significant changes in other tissues (normal-appearing WM or tumor). DTI also appears to be promising for following the response to stem cell therapies in the CNS. A study of neural progenitor cells transplanted in a mouse model after acute ischemic stroke demonstrated an increase in the FA in the ischemic boundary region, which was consistent with the formation of new axonal projections in these regions. ${ }^{114}$

\section{CONCLUSION}

In summary, DTI is a powerful method for characterizing changes in tissue microstructure associated with brain disorders. The pathologic specificity of scalar DTI measures is inadequate for a blind diagnosis, however. The use of multiple DTI measures, such as various combinations of MD, FA, $D_{\mathrm{r}}$, and $D_{\mathrm{a}}$ or the application of DTI in combination with other quantitative imaging modalities (e.g., magnetization transfer, $\mathrm{T}_{1}, \mathrm{~T}_{2}$, spectroscopy, perfusion), may help to improve the specificity of tissue pathology. In addition, the anatomic heterogeneity of the DTI measures, FA in particular, makes the interpretation of these measurements challenging. The advancement of tools for tractography, image segmentation and coregistration (between subject normalization) and anatomical templates will help to improve anatomic specificity of DTI characterization in both clinical and research settings. Finally, new diffusion measurement techniques with MRI are being developed which will improve the characterization of tissue microstructure beyond the current capabilities of DTI.

Crossing white matter tracts may be detected and resolved using HARDI/QBI methods, ${ }^{53-55,115}$ diffusion spectrum imaging, ${ }^{56,57,116}$ or new diffusion imaging models. ${ }^{17-119}$ However, in addition to detecting crossing fibers and mapping the relative orientations, it is also critical to characterize the microstructural properties of 
each fiber component to assess the effects of neuropathology. Non-monoexponential diffusion decay (e.g., fast/slow diffusion measurements) ${ }^{116,120}$ and complex models of diffusion behavior ${ }^{56,121,122}$ may also yield information that is more specific to tissue pathology. At high diffusion weighting (i.e., $b>3000 \mathrm{~s} / \mathrm{mm}^{2}$ ), the signal from restricted diffusion begins to dominate, which may be a more specific probe of microstructural components. The challenges associated with these new methods include the relatively long scan times required to acquire the hundreds of DW images and the reduced SNR of the signals at very high diffusion weighting. Vast improvements have been made in clinical DTI methodology in the past 10 years, and the next decade can be expected to lead to further important developments in CNS diffusion measurements with MRI.

Acknowledgments: The background and data for this article were generated with financial support from the Dana Foundation, the National Multiple Sclerosis Society, and National Institutes of Health grants MH62015, HD35476, and NS050466. The authors are also grateful for the collaborative contributions from $\mathrm{Yu}$-Chien $\mathrm{Wu}$ and other members of the Waisman Laboratory for Brain Imaging and Behavior.

\section{REFERENCES}

1. Basser PJ, Mattiello J, LeBihan D. Estimation of the effective self-diffusion tensor from the NMR spin echo. J Magn Reson B 1994;103:247-254.

2. Basser PJ, Mattiello J, LeBihan D. MR diffusion tensor spectroscopy and imaging. Biophys J 1994;66:259-267.

3. Conturo TE, Lori NF, Cull TS, et al. Tracking neuronal fiber pathways in the living human brain. Proc Natl Acad Sci U S A 1999;96:10422-10427.

4. Mori S, Crain BJ, Chacko VP, van Zijl PC. Three-dimensional tracking of axonal projections in the brain by magnetic resonance imaging. Ann Neurol 1999;45:265-269.

5. Basser PJ, Pajevic S, Pierpaoli C, Duda J, Aldroubi A. In vivo tractography using DT-MRI data. Magn Reson Med 2000;44: 625-632.

6. Einstein A. Investigations on the theory of the Brownian movement. New York: Dover Publications, 1956:17.

7. Le Bihan D. Molecular diffusion, tissue microdynamics and microstructure. NMR Biomed 1995;8:375-386.

8. Moseley ME, Cohen Y, Kucharczyk J, et al. Diffusion-weighted MR imaging of anisotropic water diffusion in cat central nervous system. Radiology 1990;176:439-445.

9. Chenevert T, Brunberg J, Pipe J. Anisotropic diffusion in human white matter: demonstration with MR techniques in vivo. Radiology 1990;177:401-405.

10. Norris DG. Implications of bulk motion for diffusion-weighted imaging experiments: effects, mechanisms, and solutions. J Magn Reson Imaging 2001;13:486-495.

11. Mansfield P. Real-time echo-planar imaging by NMR. Br Med Bull 1984;40:187-190.

12. Turner R, Le Bihan D, Maier J, Vavrek R, Hedges LK, Pekar J. Echo-planar imaging of intravoxel incoherent motion. Radiology 1990;177:407-414.

13. Jezzard P, Balaban RS. Correction for geometric distortion in echo planar images from B0 field variations. Magn Reson Med 1995;34:65-73.

14. Haselgrove JC, Moore JR. Correction for distortion of echoplanar images used to calculate the apparent diffusion coefficient. Magn Reson Med 1996;36:960-964.
15. Jezzard P, Barnett AS, Pierpaoli C. Characterization of and correction for eddy current artifacts in echo planar diffusion imaging. Magn Reson Med 1998;39:801-812.

16. Alexander AL, Tsuruda JS, Parker DL. Elimination of eddy current artifacts in diffusion-weighted echo-planar images: the use of bipolar gradients. Magn Reson Med 1997;38:1016-1021.

17. Reese TG, Heid O, Weisskoff RM, Wedeen VJ. Reduction of eddy-current-induced distortion in diffusion MRI using a twicerefocused spin echo. Magn Reson Med 2003;49:177-182.

18. Andersson JL, Skare S. A model-based method for retrospective correction of geometric distortions in diffusion-weighted EPI. Neuroimage 2002;16:177-199.

19. Rohde GK, Barnett AS, Basser PJ, Marenco S, Pierpaoli C. Comprehensive approach for correction of motion and distortion in diffusion-weighted MRI. Magn Reson Med 2004;51:103-114.

20. Pruessmann KP, Weiger M, Scheidegger MB, Boesiger $P$. SENSE: sensitivity encoding for fast MRI. Magn Reson Med 1999;42:952-962.

21. Alexander AL, Lee JE, Wu YC, Field AS. Comparison of diffusion tensor imaging measurements at $3.0 \mathrm{~T}$ versus $1.5 \mathrm{~T}$ with and without parallel imaging. Neuroimaging Clin N Am 2006;16: 299-309, xi.

22. Jaermann T, Crelier G, Pruessmann KP, et al. SENSE-DTI at $3 \mathrm{~T}$. Magn Reson Med 2004;51:230-236.

23. Jones DK, Williams SC, Gasston D, Horsfield MA, Simmons A, Howard R. Isotropic resolution diffusion tensor imaging with whole brain acquisition in a clinically acceptable time. Hum Brain Mapp 2002;15:216-230.

24. Shrager RI, Basser PJ. Anisotropically weighted MRI. Magn Reson Med 1998;40:160-165.

25. Papadakis NG, Xing D, Huang CL, Hall LD, Carpenter TA. A comparative study of acquisition schemes for diffusion tensor imaging using MRI. J Magn Reson 1999;137:67-82.

26. Basser PJ, Pierpaoli C. A simplified method to measure the diffusion tensor from seven MR images. Magn Reson Med 1998; 39:928-934.

27. Jones DK, Horsfield MA, Simmons A. Optimal strategies for measuring diffusion in anisotropic systems by magnetic resonance imaging. Magn Reson Med 1999;42:515-525.

28. Shimony JS, McKinstry RC, Akbudak E, et al. Quantitative diffusion-tensor anisotropy brain MR imaging: normative human data and anatomic analysis. Radiology 1999;212:770-784.

29. Hasan K, Parker DL, Alexander AL. Comparison of gradient encoding schemes for diffusion-tensor MRI. J Magn Reson Imaging 2001;13:769-780.

30. Jones DK. The effect of gradient sampling schemes on measures derived from diffusion tensor MRI: a Monte Carlo study. Magn Reson Med 2004;51:807-815.

31. Pierpaoli C, Basser PJ. Toward a quantitative assessment of diffusion anisotropy. Magn Reson Med 1996;36:893-906.

32. Alexander AL, Hasan K, Lazar M, Tsuruda JS, Parker DL. Analysis of partial volume effects in diffusion-tensor MRI. Magn Reson Med 2001;45:770-780.

33. Koay CG, Chang LC, Carew JD, Pierpaoli C, Basser PJ. A unifying theoretical and algorithmic framework for least squares methods of estimation in diffusion tensor imaging. J Magn Reson 2006;182:115-125.

34. Basser PJ, Pierpaoli C. Microstructural and physiological features of tissues elucidated by quantitative-diffusion-tensor MRI. J Magn Reson B 1996;111:209-219.

35. Conturo TE, McKinstry RC, Akbudak E, Robinson BH. Encoding of anisotropic diffusion with tetrahedral gradients: a general mathematical diffusion formalism and experimental results. Magn Reson Med 1996;35:399-412.

36. Westin CF, Maier SE, Mamata H, Nabavi A, Jolesz FA, Kikinis $R$. Processing and visualization for diffusion tensor MRI. Med Image Anal 2002;6:93-108.

37. Pierpaoli C, Jezzard P, Basser PJ, Barnett A, Di Chiro G. Diffusion tensor MR imaging of the human brain. Radiology 1996; 201:637-648

38. Ulug AM, van Zijl PJ. Orientation-independent diffusion imaging without tensor diagonalization: anisotropy definitions based on 
physical attributes of the diffusion ellipsoid. J Magn Reson Imaging 1999;9:804-813.

39. Alexander AL, Hasan K, Kindlmann G, Parker DL, Tsuruda JS. A geometric comparison of diffusion anisotropy measures. Magn Reson Med 2000;44:283-291.

40. Song SK, Sun SW, Ramsbottom MJ, Chang C, Russell J, Cross AH. Dysmyelination revealed through MRI as increased radial (but unchanged axial) diffusion of water. Neuroimage 2002;17: $1429-1436$.

41. Makris N, Worth AJ, Sorensen AG, et al. Morphometry of in vivo human white matter association pathways with diffusionweighted magnetic resonance imaging. Ann Neurol 1997;42:951962.

42. Pajevic S, Pierpaoli C. Color schemes to represent the orientation of anisotropic tissues from diffusion tensor data: application to white matter fiber tract mapping in the human brain. Magn Reson Med 1999;42:526-540.

43. Witwer BP, Moftakhar R, Hasan KM, et al. Diffusion-tensor imaging of white matter tracts in patients with cerebral neoplasm. J Neurosurg 2002;97:568-575.

44. Lazar M, Alexander AL, Thottakara PJ, Badie B, Field AS. White matter reorganization after surgical resection of brain tumors and vascular malformations. AJNR Am J Neuroradiol 2006;27:1258 1271.

45. Wu YC, Field AS, Chung MK, Badie B, Alexander AL. Quantitative analysis of diffusion tensor orientation: theoretical framework. Magn Reson Med 2004;52:1146-1155.

46. Basser PJ, Pajevic S. Statistical artifacts in diffusion tensor MRI (DT-MRI) caused by background noise. Magn Reson Med 2000; 44:41-50.

47. Mori S, Kaufmann WE, Davatzikos C, et al. Imaging cortical association tracts in the human brain using diffusion-tensor-based axonal tracking. Magn Reson Med 2002;47:215-223.

48. Catani M, Howard RJ, Pajevic S, Jones DK. Virtual in vivo interactive dissection of white matter fasciculi in the human brain. Neuroimage 2002;17:77-94.

49. Lazar M, Weinstein DM, Tsuruda JS, et al. White matter tractography using tensor deflection. Hum Brain Mapp 2003; 18:306321.

50. Stieltjes B, Kaufmann WE, van Zijl PCM, et al. Diffusion tensor imaging and axonal tracking in the human brain. Neuroimage 2001;14:723-735.

51. Wakana S, Jiang H, Nagae-Poetscher LM, van Zijl PC, Mori S. Fiber tract-based atlas of human white matter anatomy. Radiology 2004;230:77-87.

52. Jellison BJ, Field AS, Medow J, Lazar M, Salamat MS, Alexander AL. Diffusion tensor imaging of cerebral white matter: a pictorial review of physics, fiber tract anatomy, and tumor imaging patterns. AJNR Am J Neuroradiol 2004;25:356-369.

53. Tuch DS, Reese TG, Wiegell MR, Wedeen VJ. Diffusion MRI of complex neural architecture. Neuron 2003;40:885-895.

54. Frank LR. Characterization of anisotropy in high angular resolution diffusion-weighted MRI. Magn Reson Med 2002;47:10831099.

55. Alexander DC, Barker GJ, Arridge SR. Detection and modeling of non-Gaussian apparent diffusion coefficient profiles in human brain data. Magn Reson Med 2002;48:331-340.

56. Assaf Y, Basser PJ. Composite hindered and restricted model of diffusion (CHARMED) MR imaging of the human brain. Neuroimage $2005 ; 27: 48-58$.

57. Wedeen VJ, Hagmann P, Tseng WY, Reese TG, Weisskoff RM. Mapping complex tissue architecture with diffusion spectrum magnetic resonance imaging. Magn Reson Med 2005;54:13771386.

58. Hagmann P, Reese TG, Tseng WYI, Meuli R, Thirian JP, Wedeen VJ. Diffusion spectrum imaging tractography in complex cerebral white matter: an investigation of the centrum semiovale [Abstract]. Proc ISMRM 12 Kyoto 2004;623.

59. Alexander AL, Lee JE, Lazar M, et al. Diffusion tensor imaging of the corpus callosum in Autism. Neuroimage 2007;34:61-73.

60. Skare S, Andersson JL. On the effects of gating in diffusion imaging of the brain using single shot EPI. Magn Reson Imaging 2001;19:1125-1128.
61. Frank LR. Anisotropy in high angular resolution diffusionweighted MRI. Magn Reson Med 2001;45:935-939.

62. van Gelderen P, de Vleeschouwer MH, DesPres D, et al. Water diffusion and acute stroke. Magn Reson Med 1994;31:154-163.

63. Liu Y, D'Arceuil HE, Westmoreland S, et al. Serial diffusion tensor MRI after transient and permanent cerebral ischemia in nonhuman primates. Stroke 2007;38:138-145.

64. Sorensen AG, Wu O, Copen WA, et al. Human acute cerebral ischemia: detection of changes in water diffusion anisotropy by using MR imaging. Radiology 1999;212:785-792.

65. Yang Q, Tress BM, Barber PA, et al. Serial study of apparent diffusion coefficient and anisotropy in patients with acute stroke. Stroke 1999;30:2382-2390.

66. Beaulieu C, Allen PS. Determinants of anisotropic water diffusion in nerves. Magn Reson Med 1994;31:394-400.

67. Neil JJ, Shiran SI, McKinstry RC, et al. Normal brain in human newborns: apparent diffusion coefficient and diffusion anisotropy measured by using diffusion tensor MR imaging. Radiology 1998;209:57-66.

68. Suzuki Y, Matsuzawa H, Kwee IL, Nakada T. Absolute eigenvalue diffusion tensor analysis for human brain maturation. NMR Biomed 2003; 16:257-260.

69. Tyszka JM, Readhead C, Bearer EL, Pautler RG, Jacobs RE. Statistical diffusion tensor histology reveals regional dysmyelination effects in the shiverer mouse mutant. Neuroimage 2006; 29:1058-1065.

70. Song SK, Yoshino J, Le TQ, et al. Demyelination increases radial diffusivity in corpus callosum of mouse brain. Neuroimage 2005; 26:132-140.

71. Harsan LA, Poulet P, Guignard B, et al. Brain dysmyelination and recovery assessment by noninvasive in vivo diffusion tensor magnetic resonance imaging. J Neurosci Res 2006;83:392-402.

72. Sun SW, Liang HF, Trinkaus K, Cross AH, Armstrong RC, Song SK. Noninvasive detection of cuprizone induced axonal damage and demyelination in the mouse corpus callosum. Magn Reson Med 2006;55:302-308.

73. Henry RG, Oh J, Nelson SJ, Pelletier D. Directional diffusion in relapsing-remitting multiple sclerosis: a possible in vivo signature of Wallerian degeneration. J Magn Reson Imaging 2003;18:420 426.

74. Choi SJ, Lim KO, Monteiro I, Reisberg B. Diffusion tensor imaging of frontal white matter microstructure in early Alzheimer's disease: a preliminary study. J Geriatr Psychiatry Neurol 2005;18:12-19.

75. Assaf Y, Ben-Sira L, Constantini S, Chang LC, Beni-Adani L. Diffusion tensor imaging in hydrocephalus: initial experience. AJNR Am J Neuroradiol 2006;27:1717-1724.

76. Concha L, Gross DW, Wheatley BM, Beaulieu C. Diffusion tensor imaging of time-dependent axonal and myelin degradation after corpus callosotomy in epilepsy patients. Neuroimage 2006; 32:1090-1099.

77. Gross DW, Concha L, Beaulieu C. Extratemporal white matter abnormalities in mesial temporal lobe epilepsy demonstrated with diffusion tensor imaging. Epilepsia 2006;47:1360-1363.

78. Moeller FG, Hasan KM, Steinberg JL, et al. Diffusion tensor imaging eigenvalues: preliminary evidence for altered myelin in cocaine dependence. Psychiatry Res 2007;154:253-258.

79. Tievsky AL, Ptak T, Farkas J. Investigation of apparent diffusion coefficient and diffusion tensor anisotropy in acute and chronic multiple sclerosis lesions. AJNR Am J Neuroradiol 1999;20: 1491-1499.

80. Werring DJ, Clark CA, Barker GJ, Thompson AJ, Miller DH. Diffusion tensor imaging of lesions and normal-appearing white matter in multiple sclerosis. Neurology 1999;52:1626-1632.

81. Field AS, Alexander AL. Diffusion tensor imaging in cerebral tumor diagnosis and therapy. Top Magn Reson Imaging 2004;15: 315-324.

82. Laundre BJ, Jellison BJ, Badie B, Alexander AL, Field AS. Diffusion tensor imaging of the corticospinal tract before and after mass resection as correlated with clinical motor findings: preliminary data. AJNR Am J Neuroradiol 2005;26:791-796. 
83. Gauvain KM, McKinstry RC, Mukherjee P, et al. Evaluating pediatric brain tumor cellularity with diffusion-tensor imaging. AJR Am J Roentgenol 2001;177:449-454.

84. Guo AC, Cummings TJ, Dash RC, Provenzale JM. Lymphomas and high-grade astrocytomas: comparison of water diffusibility and histologic characteristics. Radiology 2002:224:177-183.

85. Beppu T, Inoue T, Shibata Y, et al. Measurement of fractional anisotropy using diffusion tensor MRI in supratentorial astrocytic tumors. J Neurooncol 2003;63:109-116.

86. Beppu T, Inoue T, Shibata $\mathrm{Y}$, et al. Fractional anisotropy value by diffusion tensor magnetic resonance imaging as a predictor of cell density and proliferation activity of glioblastomas. Surg Neurol 2005;63:56-61.

87. Lu S, Ahn D, Johnson G, Law M, Zagzag D, Grossman RI. Diffusion-tensor MR imaging of intracranial neoplasia and associated peritumoral edema: introduction of the tumor infiltration index. Radiology 2004;232:221-228.

88. Tropine A, Vucurevic G, Delani P, et al. Contribution of diffusion tensor imaging to delineation of gliomas and glioblastomas. J Magn Reson Imaging 2004;20:905-912.

89. Tsuchiya K, Fujikawa A, Nakajima M, Honya K. Differentiation between solitary brain metastasis and high-grade glioma by diffusion tensor imaging. Br J Radiol 2005;78:533-537.

90. Morita K, Matsuzawa H, Fujii Y, Tanaka R, Kwee IL, Nakada T. Diffusion tensor analysis of peritumoral edema using lambda chart analysis indicative of the heterogeneity of the microstructure within edema. J Neurosurg 2005;102:336-341.

91. Yung A, Poon G, Qiu D, et al. White matter volume and anisotropy in preterm children: A pilot study of neurocognitive correlates. Pediatr Res 2007 [Epub ahead of print].

92. Muller MJ, Greverus D, Weibrich C, et al. Diagnostic utility of hippocampal size and mean diffusivity in amnestic MCI. Neurobiol Aging 2007;28:398-403.

93. Alexopoulos GS, Kiosses DN, Choi SJ, Murphy CF, Lim KO. Frontal white matter microstructure and treatment response of late-life depression: a preliminary study. Am J Psychiatry 2002; 159:1929-1932.

94. O’Sullivan M, Morris RG, Huckstep B, Jones DK, Williams SC, Markus HS. Diffusion tensor MRI correlates with executive dysfunction in patients with ischaemic leukoaraiosis. J Neurol Neurosurg Psychiatry 2004;75:441-447.

95. Holtmannspotter M, Peters N, Opherk C, et al. Diffusion magnetic resonance histograms as a surrogate marker and predictor of disease progression in CADASIL: a two-year follow-up study. Stroke 2005;36:2559-2565.

96. Wardlaw JM, Keir SL, Bastin ME, Armitage PA, Rana AK. Is diffusion imaging appearance an independent predictor of outcome after ischemic stroke? Neurology 2002;59:1381-1387.

97. Stinear CM, Barber PA, Smale PR, Coxon JP, Fleming MK, Byblow WD. Functional potential in chronic stroke patients depends on corticospinal tract integrity. Brain 2007;130:170-180.

98. Lin X, Tench CR, Morgan PS, Niepel G, Constantinescu CS. 'Importance sampling' in MS: use of diffusion tensor tractography to quantify pathology related to specific impairment. J Neurol Sci 2005;237:13-19.

99. Yu CS, Li KC, Xuan Y, Ji XM, Qin W. Diffusion tensor tractography in patients with cerebral tumors: a helpful technique for neurosurgical planning and postoperative assessment. Eur J Radiol 2005;56:197-204.

100. Powell HW, Parker GJ, Alexander DC, et al. MR tractography predicts visual field defects following temporal lobe resection. Neurology 2005;65:596-599.

101. Mamata Y, Mamata H, Nabavi A, et al. Intraoperative diffusion imaging on a 0.5 Tesla interventional scanner. J Magn Reson Imaging 2001;13:115-119.

102. Nimsky C, Ganslandt O, Hastreiter P, et al. Intraoperative diffusion-tensor MR imaging: shifting of white matter tracts during neurosurgical procedures--initial experience. Radiology $2005 ; 234: 218-225$.
103. Nimsky C, Ganslandt O, Merhof D, Sorensen AG, Fahlbusch R. Intraoperative visualization of the pyramidal tract by diffusion-tensor-imaging-based fiber tracking. Neuroimage 2006;30:1219-1229.

104. Salvan CV, Ulmer JL, Mueller WM, Krouwer HG, Prost RW, Stroe GO. Presurgical and intraoperative mapping of the motor system in congenital truncation of the precentral gyrus. AJNR Am J Neuroradiol 2006;27:493-497.

105. Wakamoto H, Eluvathingal TJ, Makki M, Juhasz C, Chugani HT. Diffusion tensor imaging of the corticospinal tract following cerebral hemispherectomy. J Child Neurol 2006;21:566-571.

106. Wieshmann UC, Symms MR, Clark CA, et al. Wallerian degeneration in the optic radiation after temporal lobectomy demonstrated in vivo with diffusion tensor imaging. Epilepsia 1999;40: $1155-1158$.

107. Khong PL, Kwong DL, Chan GC, Sham JS, Chan FL, Ooi GC. Diffusion-tensor imaging for the detection and quantification of treatment-induced white matter injury in children with medulloblastoma: a pilot study. AJNR Am J Neuroradiol 2003;24:734740.

108. Qiu D, Leung LH, Kwong DL, Chan GC, Khong PL. Mapping radiation dose distribution on the fractional anisotropy map: applications in the assessment of treatment-induced white matter injury. Neuroimage 2006;31:109-115.

109. Sundgren PC, Fan X, Weybright P, et al. Differentiation of recurrent brain tumor versus radiation injury using diffusion tensor imaging in patients with new contrast-enhancing lesions. Magn Reson Imaging 2006;24:1131-1142.

110. Mabbott DJ, Noseworthy MD, Bouffet E, Rockel C, Laughlin S. Diffusion tensor imaging of white matter after cranial radiation in children for medulloblastoma: correlation with IQ. Neurooncology 2006;8:244-252.

111. Khong PL, Leung LH, Fung AS, et al. White matter anisotropy in post-treatment childhood cancer survivors: preliminary evidence of association with neurocognitive function. J Clin Oncol 2006; 24:884-890.

112. Blood AJ, Tuch DS, Makris N, Makhlouf ML, Sudarsky LR, Sharma N. White matter abnormalities in dystonia normalize after botulinum toxin treatment. Neuroreport 2006;17:1251-1255.

113. Sinha S, Bastin ME, Wardlaw JM, Armitage PA, Whittle IR. Effects of dexamethasone on peritumoural oedematous brain: a DT-MRI study. J Neurol Neurosurg Psychiatry 2004;75:16321635.

114. Jiang Q, Zhang ZG, Ding GL, et al. MRI detects white matter reorganization after neural progenitor cell treatment of stroke. Neuroimage 2006;32:1080-1089.

115. Khachaturian MH, Wisco JJ, Tuch DS. Boosting the sampling efficiency of q-Ball imaging using multiple wavevector fusion. Magn Reson Med 2007;57:289-296.

116. Wu Y-C, Alexander AL. Hybrid diffusion imaging. Neuroimage 2007 March 24 [Epub ahead of print].

117. Peled S, Friman O, Jolesz F, Westin CF. Geometrically constrained two-tensor model for crossing tracts in DWI. Magn Reson Imaging 2006;24:1263-1270.

118. Anderson AW. Measurement of fiber orientation distributions using high angular resolution diffusion imaging. Magn Reson Med 2005;54:1194-1206.

119. Behrens TE, Berg HJ, Jbabdi S, Rushworth MF, Woolrich MW. Probabilistic diffusion tractography with multiple fibre orientations: What can we gain? Neuroimage 2007;34:144-155.

120. Mulkern RV, Gudbjartsson H, Westin CF, et al. Multi-component apparent diffusion coefficients in human brain. NMR Biomed 1999;12:51-62.

121. Bennett KM, Schmainda KM, Bennett RT, Rowe DB, Lu H, Hyde JS. Characterization of continuously distributed cortical water diffusion rates with a stretched-exponential model. Magn Reson Med 2003;50:727-734.

122. Jensen JH, Helpern JA, Ramani A, Lu H, Kaczynski K. Diffusional kurtosis imaging: the quantification of non-Gaussian water diffusion by means of magnetic resonance imaging. Magn Reson Med 2005;53:1432-1440. 